\title{
An Activity Classification for Vibrotactile Phenomena
}

\author{
Conor O'Sullivan ${ }^{1}$ and Angela Chang ${ }^{2}$ \\ 1600 North US Highway 45, DS-175, Libertyville, IL 60048, USA \\ conor.o'sullvan@motorola.com \\ 20 Ames St. Cambridge, MA 02139 USA \\ anjchang@media.mit.edu
}

\begin{abstract}
We observe that the recent availability of audio-haptic actuators allow richer vibration content to be available in commercial devices. However, we note that consumers are unable to take advantage of these rich experiences, mainly due to the lack of a descriptive language for vibration. We analyze the current methods for classifying vibrations. We propose a new framework for describing vibrotactile haptic phenomena, based on an organizing the media based on content activity. We describe this naming system, based on Russolo's families of noise, and address other pertinent issues to introducing vibration content into commercial devices.
\end{abstract}

\section{Introduction}

Today there is a gap in the community and industry of haptic actuation technology (specifically vibrotactile sensations) that needs to be addressed to allow a more general adoption of and dedicated engagement with the technology. In various forums of research, presentation and informal discussion it was found that although the average consumer or target adopter of such technology seems comfortable or even enthusiastic with their experience of haptics, the extent to which they are able to communicate and describe this experience seems very limited. The language of describing haptic phenomena in consumer electronic devices seems thus far limited to a small selection of words such as 'buzz' or 'vibrate', even as the devices are evolving to allow richer experiences (such as gesture input, vibration enhanced ringtones, and UI feedback).

To understand how a general population can talk about haptics they need to understand how to create and distinguish among haptic effects. The correlation between audio and haptics in the case of the mobile device has already been shown [6]. Audio-haptics (driving vibrations using audio signals [27]) for consumer devices can be generated by audio manipulation and synthesis [15], but are perceived tactilely as a more advanced form of vibration. It is of course sufficient to speak of vibe or buzzing in the general case of vibration alert mechanisms. Given the range and depth of current and future haptic actuation technologies however, it is imperative that a more user-friendly, sensorial descriptions are available.

This will allow the general consumer to become knowledgeable, interested and proficient in the language of haptic description. A consumer armed with such proficiency 
and enthusiasm can only serve to drive interest and consequently propagate the development of the technology and of design. This type of language can develop from new or existing forms of haptic engagement, but the industry may be better served in the medium term to associate with 'real world' or ecologically familiar constructs.

\section{Motivation for Describing Vibrotactile Phenomena}

Haptics is the science of touch, encompassing both kinesthetic and tactile phenomena. The study of haptics is a wide field, some areas of research are: 3D force-feedback actuation technologies, perception of touch (psychophysics), tactile display of shapes and textures, temperature sensing, and awareness and location relationships between body parts. Because of the broad applications of haptics, terminology between different subsections of haptic research are usually specific to their sub disciplines, and may not apply to other areas of research (e.g. perception measurements of vibrotactile displays might not apply to 3D force feedback displays).

For the purposes of this paper, we refer to the specific phenomenon of vibrotactile stimulation (or vibes), tactile stimulation using vibration. We address this particular area due to the recent availability of better vibration actuators in consumer products that allow for greater control over tactile sensation [6, 19]. Increased resolution and variation enables more dynamic range in the vibration content. This new expressiveness could be used as a selling point for differentiating haptic products. The featured improvement of haptic resolution could be appreciated, providing consumers could differentiate among complex vibrotactile stimuli.

\subsection{Toward a Generalized Knowledge of Haptics}

The combination of temporal and spatial information provided by haptics to reinforce perception and communication is ubiquitous. For many years, vibration in commercially available mobile devices, such as pagers and mobile phones, alerted people of calls or messages. The ubiquity of vibrating alerts in mobile devices has also misled many consumers to believe haptics is equivalent to vibration. Furthermore, when the authors demonstrated a vibration-enhanced mobile phone to novice users, general comments received about haptics provide an illustrative example of the lack of public haptic awareness and vocabulary.

There appeared to be a large vocabulary gap between the scientific and HCI community and haptics understanding in the common usage. Quality in consumer products often relates directly to touch (weight, surface finish, and contours). However, there is little vocabulary to describe haptic sensations, in particularly vibrations. When asked to describe haptic quality, a surprising number of people would relate haptic vibrations to audio [6]. Many people simply said "it feels different", but were unable to say why.

This lack of expressiveness about haptics is surprisingly minimal in contrast with that of the sense of smell. Before the days of aromatherapy, there was little conscious awareness of the cause and effect of smell. In fact, until marketing began to become popular, body odor was not considered a problem [18]. People did not typically describe smells, nor did they obsess about altering them. Nowadays, people can identify 
common scents in products (e.g. cinnamon and vanilla) and they can also understand smells can be controlled and distinguish differences between smells [1]. The common vocabulary for smells has increased from merely "strong or noxious" to a myriad of descriptive phrases "lavender, rosemary, bergamot, etc". Furthermore, there is a vast amount of consumer interest in creating scents (one can easily find recipes online for air fresheners, relaxation, and medical therapy). The large perfume market gives evidence to the fact that "smell is a commodity" [18, p. 62]. We are equally interested in how to create enlightened tactile consumers, a tacterati, so to speak.

\subsection{Raise Haptic Consciousness}

One way to raise consciousness is to create objects that stimulate the haptic senses more directly. Various force-feedback and vibration gaming joysticks have incorporated haptics sensations for years. Certain phones include haptics as a feature inherent in audio quality. Digitally controlled haptics is subtly integrating into consumer products, but it is still very hard for consumers to distinguish and disseminate haptic sensations. Could this lack of definition be due to the dearth of vocabulary to describe haptic qualities in our culture?

The visualization of haptics might be a challenge because of the lack of awareness of haptic phenomenon in popular culture. It is rare to find language for distinguishing the roughness of textures from one another (e.g. dotted pattern versus serrated rows). There are few common ways to describe vibration profiles (sawtooth versus sine waves). For example, try to describe the difference in tremors in the floor due to heating systems "oscillations", traffic "rumblings" and earthquakes "shockwaves". Even among researchers in haptics, there are few generalized terms to describe force profiles or vibration envelopes.

A quick survey of the consumer marketplace finds that many haptic experiences are targeted to the high-end of consumer product lines. Force feedback joysticks are high-priced and geared toward the gaming public. Devices touted for their tactile qualities, such as high-end audio equipment, are more expensive than offerings for the general public. In most cases, devices displaying greater vibration control are targeted to special-needs users [2]. The target market for haptic devices seems to be very specialized and narrow.

Perhaps social consciousness will be raised when product literature incorporates descriptive expressions for haptic information that can be repeated and discussed by the general population. Once the public can express the value of haptic sensations, haptic visualization may be more common, and haptics might become a commodity by which differentiates products.

\subsection{Haptics in Infancy Parallels Sonic Development and Language}

We propose a lexicon beyond buzzes, pulses, and shaking of the pager motor era. Given that the lexicon of haptic description is in its infancy, a correlation may be drawn with the industry of olfaction around aromatherapy (as previously described) or perhaps general electronic audio in the first half of the last century: 


\subsubsection{Composers and Theorists}

As early instruments such as the Telharmonium began to appear, people such as Ferrucio Busoni saw a role for such machinery in the future synthesis of music and sound. This was acted on more expressly by the Futurist movement and artists such as Luigi Russolo, who realized the musical, sonic and social value of noise [20]. Russolo began to develop a language of sound and of noise, expressing his categorization of noise into six categories. This will be looked at later, as an example of a basis for haptic expression.

Edgard Varese was another artist who approached music as an instantiation of sound and consequently looked to expand the range of techniques of synthesis, crying out for electronic synthesis technology until it became available practically, to realize his auditory design. The work of such pioneers and others including Schaeffer and Stockhausen, allowed the language and technique of electronic audio to emerge [26].

\subsubsection{Auditory Interface Design}

More recently work has taken place that looks at auditory interface design and how the perception and expression of such feedback can be realized. Gaver and others speak of ecological acoustic perception, to enable a user to associate sounds in the natural environment to the presentation of information from a computer interface [21, 22]. Blattner, Brewster and others have looked at earcons, or more musical motives, as a means of presenting information that has associated meaning either inherently or learned over time $[23,24,25]$.

\subsubsection{Haptic Vibrations Are Easy to Make, but Not Easy to Talk About}

Currently in mobile devices the types of auditory feedback we experience includes voice, ringtones, music playback, user interface (UI) sounds and gaming. Among these types and even among UI sounds, we hear examples of both earconography and auditory iconography. Haptics in today's mobile devices where it occurs beyond basic alerting vibe mechanism is often mapped to the rhythm of the sound and so tends to exist as derivatives of both categories of sound feedback. It has been shown previously how haptics can be generated with and from sounds. Haptics therefore can be relatively straightforward to produce but as we've seen are not always so easy to discuss.

\subsection{Study of Classifications for Tactile Phenomena}

We present two examples of the benefits of tactile classification below, haptic exploratory procedures and tactile language displays.

\subsubsection{Haptic Exploratory Procedures}

The haptic exploratory procedures (EPs) demonstrate how touch can be both an active and passive way of interpreting information [13]. This classification of EPs has allowed researchers to generalize haptic procedures, and better understand the mechanical actions performed by our hands. Additionally, by organizing the knowledge gained from touch activities, we can compare haptics with other informationgathering modalities. Table 1 summarizes some qualities of haptics relevant to information gathering. 
Table 1. Comparison of haptics attributes to audition and vision

\begin{tabular}{|c|c|c|c|}
\hline & Touch & Audition & Vision \\
\hline $\begin{array}{l}\text { Information } \\
\text { given relative to } \\
\text { person }\end{array}$ & $\begin{array}{l}\text { Useful for details } \\
\text { smaller than a person }\end{array}$ & $\begin{array}{l}\text { Useful for gauging } \\
\text { distance relative to a } \\
\text { person }\end{array}$ & $\begin{array}{l}\text { Useful for gauging } \\
\text { size and distance } \\
\text { relative to a person }\end{array}$ \\
\hline $\begin{array}{l}\text { Temporal } \\
\text { aspects }\end{array}$ & \multicolumn{2}{|c|}{$\begin{array}{l}\text { Identification by parts. Information is gathered } \\
\text { by sensing information over time. }\end{array}$} & $\begin{array}{l}\text { Vision uses both part } \\
\text { and whole images to } \\
\text { identify whole things }\end{array}$ \\
\hline $\begin{array}{l}\text { Active and } \\
\text { Passive Identi- } \\
\text { fication }\end{array}$ & \multicolumn{2}{|c|}{$\begin{array}{l}\text { Active and Passive methods for information } \\
\text { gathering (e.g. applying pressure or echoloca- } \\
\text { tion) }\end{array}$} & Passive only \\
\hline Spatial aspects & $\begin{array}{l}\text { One-to-one only, a } \\
\text { close proximity sen- } \\
\text { sory experience }\end{array}$ & \multicolumn{2}{|c|}{ Can be close or remote, can be broadcast } \\
\hline $\begin{array}{l}\text { Illusory Possi- } \\
\text { bilities }\end{array}$ & Touch is hard to fake & \multicolumn{2}{|c|}{$\begin{array}{l}\text { Easy to represent, replicate, reproduce digi- } \\
\text { tally }\end{array}$} \\
\hline
\end{tabular}

By understanding the classification of EPs, we can describe how physical details are found by certain hand motions

\subsubsection{Tactile Language Displays}

Existing tactile languages can be subdivided into two classes of languages, alphabetic and symbolic. The first class, alphabetic language uses the representation of the alphanumeric letters to form words. Examples are chording keyboards, Braille, Moon, and telegraphs. The second class, symbolic language, represents higher-level concepts that are not mapped directly to words, but rather, abstract ideas and expressive emotions. Examples of symbolic language are facial expressions, hand gestures and body language for expressing interest and emotional state. Symbolic and alphabetic language can be combined in a language, such as in Morse code and fingerspelling. The combined methods enable users to transmit information more quickly than working with either method alone. Fingerspelling is more expressive than Morse code, using gestures made by one hand to rest on another hand, making full use of the EPs. Fingerspelling has faster transmission rates than Morse code. Sign language displays, when used with vision, are commonly used in public speeches because of the broadcast nature and quick speed of transmission.

In terms of vibrotactile displays, Tadoma allows users to receive intonation information directly from the vibration of a speaker's throat [16]. Vibrotactile devices, e.g. Tactaid, remap ambient audio frequencies to vibration channels. Combining vibration signals with visual cues allow faster transmission of information than the hand signing or alphabetic methods. Vibrotactile displays allow for quick transmission, but are private rather than broadcast. The unobtrusiveness of these displays has been employed in vibrotactile arrays to provide navigational and geographic information [11] and entertainment [9].

Classification schemes give information for comparison among groups of related items, visibility of differences between items, and give us a way to control communications about different subsets [28]. By classifying the tactile languages, comparisons 
can be made between the transfer rates of alphabetic languages and symbolic languages. Among researchers, there have been previous approaches to describe vibrotactile content. The need for a flexible, yet structured classification scheme becomes even more necessary as vibes are now mapped to buttons, ringtones, and other UI media in commercial devices. We present our experience using the following classification methods while working with audio-haptics for commercial products.

\section{Example Descriptive Frameworks for Vibrotactile Phenomena}

\subsection{Descriptions of Vibes Using Compositional Elements}

Like sound, the compositional elements of any vibration can be described by frequency, intensity, duration, waveform and space. The first method we tried for describing vibrations was to create vibration files from the compositional elements of frequency, envelope, and waveform shapes (sine, triangle, and square waves). The skin is most perceptive of intensity, duration and waveform, and many variations of these elements were created to create a library of haptic waveforms.

From testing of elements in the library, we confirmed much of the psychophysics testing that there was little correlation between a perceived "pitch" and frequency (unlike in audio). High frequencies $(>250 \mathrm{~Hz}$ ) have higher pitches and feel smooth, while lower frequencies (around 100-150 Hz) sound like buzzes and feel rough. In general, a sine wave was perceived as "smoother" than a square wave. Gunther compares the variations of waveform to the "texture" of tactile stimuli [9]. Varying the waveform envelope was compared to adjusting the volume of a sound. The intensity of entrance and exit of the waveform, respectively called the attack and release angle. Sharper attacks were perceived as pulses, while smoother attacks eased the vibe into the users' focus of attention. Similarly, smooth releases were perceived as "fading away".

In all, although many hundreds of combinations were created in the laboratory for testing, it was not clear whether users were able to name a particular sensation. The differences between two similar compositions (e.g. $100 \mathrm{~Hz}$ vs. $110 \mathrm{~Hz}$ ) were oftentimes indistinguishable to the end user. Users were able to distinguish waveforms of different envelopes much easier than the other compositional elements, but with names like " $100 \mathrm{~Hz}$ sine wave fading for 0.5 seconds", though helpful for a sound designer, were too technical for an end-user.

\subsubsection{Rhythm and Tempo Descriptors}

Brewster, et.al, has experimented with applying musical techniques for varying the waveform envelopes in tactile icon design [4,24]. In their work, subtlety and variation are emphasized, along with integration with a user interface. However, the problem with assigning too many vibrations to an interface is that the skin can become overloaded, much like the ears. One way to distinguish vibrations was by varying rhythm and tempo. References to vibrations with "slow soft tempo" or "dot-dot-dash" using the Morse code-like descriptions are common in literature on vibration devices (e.g. haptic mouse textures[29]).

Rhythm and tempo descriptions are a more user-friendly way of describing haptic actuation than describing the compositional elements. One drawback of this technique is these descriptions are still abstract to the end-user. A user might not be familiar 
with the terminology of musical or rhythmic classifications, and further, the terminology is still symbolic of the vibration media. Issues of interest to us were "how does a car crash feel?" or "what should a button press experience be?"

\subsubsection{Form Factor Based Descriptions}

One main problem of vibrotactile simulations is that there can be a variation of form factors and arrangements of actuators. Various researchers have explored a vast number of ergonomic shapes (e.g. knobs, mice, phones, joysticks) and surfaces (e.g. rollers, vests, chairs, human bodies) for tactile stimulation $[3,7,8,12,14,17,30]$. Some additional physical language needs to be used to describe vibrations from devices with specific form factors. If more than single-point vibration is used, spatially oriented words such as "diagonal" "across" or "up down" are appropriate. One difficulty with describing vibrations on these form-factor descriptors is that there is a critical reliance on the exact implementation of the vibration.

\subsubsection{Descriptions Used When Combined with Other Modalities}

When combined with other modalities, it is usual for the descriptions of the haptic phenomena to be associated with the other modality. When spoken conversation is augmented with a vibrotactile channel, there are tactile gestures that mimic spoken interactions [5]. By sampling vibrations off of surface textures [14] or existing audio, users were able to attribute the surface or audio information to the vibration. For example, users can say, "I like the feel of that rough surface". When testing a vibration-enhanced motorcycle driving game, designers sampled the audio from a real motorcycle, the result was that the "motorcycle sound feels realistic".

In ActiveTV, O'Modhrain found that there was ambiguity of what character was associated with haptic content when there were many actors on a screen[31]. For example, if an animal kicked a ball into a tree, would the vibration represent the animal's foot kicking the ball, the ball bouncing, or the impact between the ball and the tree? These lessons suggested to us that vibrations might be described by activity.

\subsubsection{Design Criteria for a New Vibration Classification Scheme}

Though technically descriptive the prior methods described technical information (such as compositional elements and musical terms), or relied heavily on a particular form factor, which was not transferable to other devices, resulting in problems scaling the media across different hardware platforms. The last method of using language from another modality seemed to amplify the awareness of other modalities, but did not make users more aware of how to describe their haptic sensations. We believed that in order to bring vibration awareness to the forefront, one possibility was to use more activity-driven language to describe these vibrations.

\section{Proposed Activity Classification for Vibes}

\subsection{Translating Sound and Vibe}

Since haptics and sound co-exist on our mobile devices today it may be useful to look at the development of the language of sound and see how this can be applied to a new development of a language in haptics. One way to do this is to observe some of the 
Table 2. Russolo's families of noise and a haptic activity classification derivative

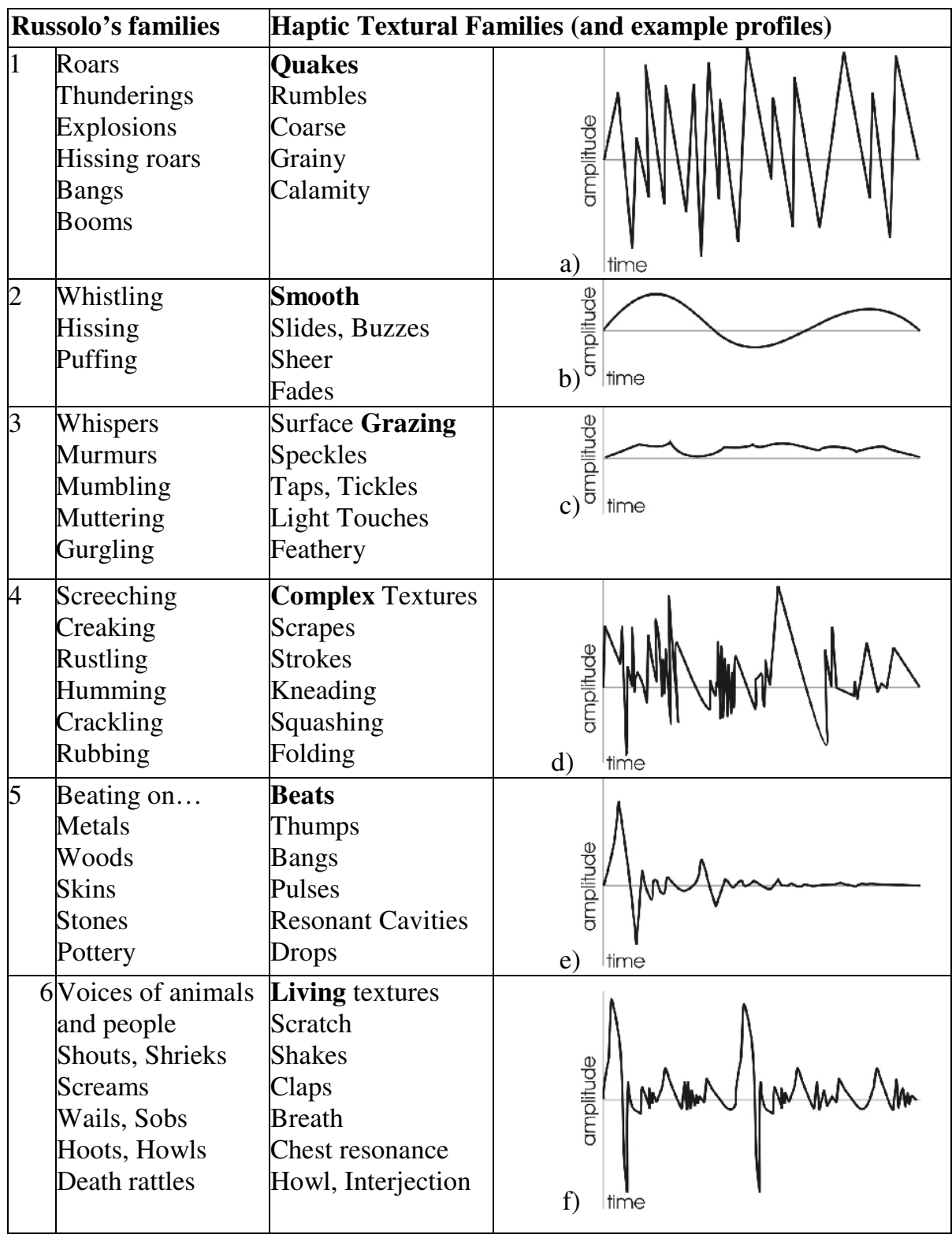

Fig. 1(a-f). Typical sound profiles for the 6 classes of activities

thinking around the beginnings of the electronic sound movement and see what parallels, if any, can be drawn. Luigi Russolo, one of the early proponents of music as an instance of noise, published his manifesto in 1913 describing families of noise he 
considered groups that were fundamental in noise [20]. Here we look at these and show how a similar grouping categorization can be derived and applied to haptic textures. This is intended to serve as a basic framework by which a haptic language could develop. It is not intended to be a definitive list of haptic textures, but rather a starting point for thought and further structure to grow. Table 1 shows our proposed classification method for haptic vibrations.

\subsection{Sample Vibe Classification and Waveform Shapes}

To explore more fully some ecological examples of these haptic textures, it is useful to apply real world examples such as

Group One, Quakes: Riding a bicycle over tracks

Group Two, Smooth: Ice Skating

Group Three, Grazing: Goosebumps

Group Four, Complex: Picking up an object, interacting with the object

Group Five, Beats: Bang on the drum, a tap on the shoulder

Group Six, Living: Feeling resonance in your chest

The textures of groups 4 and 6 are related and could perhaps together form a wider group. They comprise the group of textures that make up a large part of our daily haptic experiences. All these groups can be represented visually as audio-haptic waveform representations as shown in figures 1a-1e. Quakes typically have coarse textures, large amplitudes, and sustained energy oscillations (fig. 1a). Smooth textures tend to have small changes in amplitude (fig. 1b). Grazing vibes are characterized by low amplitudes, perhaps at the thresholds of perception (fig. 1c). Complex textures encompass the wide range of activities where impulses and noise compete for attention. Beats are impulse-driven, quickly damped resonances (fig.1e). Living vibes are characterized by their periodic nature (fig.1f).

By using these classifications, we can lump different activities with typical profiles, and find that within the groups, substitutions in waveforms can be made. In answer to the previous question, "what should a button press experience be?" our answer in to use media from group 5 , highly damped impulses. When a vibe is needed for moving a pointer across a screen, we can select from smooth (group 2) when there is nothing of interest and then use grazing textures (group 3) when they are over a button. Similarly, when the system has a notification message, it may be appropriate to either use a beat texture (group 5) or a start-up or power-down quake (group 1). For some ringtones, or subtle alerts, we may use living textures under audio (group 6) or quakes, if the situation calls for increased stimulation.

\subsection{Ecological and Musical Considerations}

Gaver lays out a framework for everyday listening that can provide a similar method for categorizing haptic textures. Gaver groups sounds firstly by Liquid, Solid and Gas categories and their Hybrid crossovers [21]. There are then further sub-groups given within each category. Some of these sub-groups seem immediately suited to haptic groups, such as deformation, impact, scraping and indeed we see these sound types have similarities to some of the haptic groups above. Similarly, a mapping of the earcon motive elements to the specific case of haptic feedback may be given by looking 
at features such as rhythm, timbre, and dynamics. These features can contribute to a haptic language by exploring their equivalents (or comparables) like rhythm, texture and strength.

\subsection{Tools and Methods for Creating Haptic and Describing Phenomenon}

It has been shown previously that textures can be explored and created for consumer electronic devices, such as the mobile device, that exist in the marketplace today. Though the resolution required to synthesize the feeling of some of these textures described above doesn't exist currently in mass market products, a broad range of effects and even a good sample set from each of these families can be created using tools already employed by any sound designer.

In our experience, having an activity based classification scheme works a bit better than the previous schemes. When developing vibes among a group of haptic novices, we have found that discussing "rumbles, slides, taps, scrapes, pulses, and echoes" helps make it easier for users, marketing people, and content designers to distinguish among groups of profiles. Classes of content (e.g. beats) are used among different form factors and UI elements (e.g. different buttons) because the profiles provide transparency into the types of sensations within a class. The descriptors seem to give visibility to the vibrations better than prior methods.

We are aware that with any classification scheme, there is a tradeoff between being too vague and too specific. Although technical information is not specified, the activity vibration classification seems to give developers and users some control to manage content relating to the different profile groups. Of course, more refinement may be needed as people become more familiarity with the sensations.

\section{Conclusion}

A general issue for haptic visualization is that there is currently little consumer understanding of haptic phenomenon. Further work will have to be done to raise the public consciousness and educate the world about understanding and describing the sense of touch. In this paper we proposed a new approach to classify vibrations, an activity classification scheme, influenced by the classification schemes of music. This approach is a more sensorial description and we hope, will be more descriptive to end-users.

Of course, the real test of the approach to framing a common language for haptic expression is its ease and success of adoption by the general 'haptic consumer'. The approach described herein seems useful and intuitive to the authors, but it remains to be seen how the consumer adapts to the taxonomy. The burden is on the promoters of the science not only to adopt this or similar methods but to reinforce the terminology and language so that it is readily associated with the senses. This can be achieved through consistent use over time, persistence and the penetrative force of marketing.

The ability to discuss, distinguish, and judge haptic phenomenon introduces users to the potential richness of these sensations. We also hope that once a framework for describing haptic effects is commonly employed, there might be interest and opportunity for users to create and share their own haptic effects. 


\section{References}

[1] Ackerman, D; A natural history of the senses, New York: Random House, 1990, p.80.

[2] Auer, E.T., Jr, and Bernstein, L.E. Temporal and spatio-temporal vibrotactile displays for voice fundamental frequency: An initial evaluation of new vibrotactile speech perception aid with normal-hearing and hearing-impaired individuals. Journal of Acoustical of Society of America. 104, 4. October1998, 2477-2489.

[3] Brave, S. and Dahley, A., inTouch: A Medium for Haptic Interpersonal Communication, Extended Abstracts of CHI 1997, ACM Press (1997), 363-364.

[4] Brewster, S. A., Wright, P. C., and Edwards, A. D. N., "Experimentally derived guidelines for the creation of earcons," in Proc. of HCI'95, Huddersfield, UK, 1995.Burdea, Grigore C. Force and Touch Feedback for Virtual Reality, John Wiley \& Sons; New York, 1996.

[5] Chang, A., O'Modhrain, M. S., Jacob, R. J., Gunther, E., Ishii, H. ComTouch: design of a vibrotactile communication device. Proceedings of Designing Interactive Systems 2002, ACM Press(2002), 312-320.

[6] Chang, A. and O'Sullivan, C. Audio-Haptic Feedback in Mobile Phones. CHI Extended Abstracts 2005, ACM Press (2005), 1264-1267.

[7] Geldard, F., and Sherrick, C., The cutaneous rabbit: A perceptual illusion, “ Science, 178, 1972, 178-179.

[8] Geldard, F.A. Body English, Random House, 1967.

[9] Gunther, E. (2001). Skinscape: A Tool for Composition in the Tactile Modality. MIT MSEE Thesis, 2001.

[10] Ishii, H. and Ullmer, B. Tangible Bits: Toward Seamless Interfaces between People, Bits and Atoms, Proc. of CHI 1997, ACM Press (1997), 234-241.

[11] Jones, L., Nakamura, M. and Lockyer, B. Development of a tactile vest, Proc. of Haptic Symposium 2004, IEEE Press (2004), 82-89.

[12] Lécuyer, A., Burkhardt, J.M., and Etienne, L. Feeling bumps and holes without a haptic interface: the perception of pseudo-haptic textures, Proc. of CHI 2004, ACM Press (2004), 239-246.

[13] Lederman, S. J. and Klatzky, R. L. Hand Movements: A Window into Haptic Object Recognition, Journal of Cognitive Psychology; 19, 3, pp. 342-368, 1987.

[14] Minsky, M. D. R. Computational Haptics: The Sandpaper System for Synthesizing Texture for with a Force-Feedback Haptic Display. MIT PhD Thesis, 1995.

[15] O'Sullivan, C. and Chang, A. Dimensional Design; Explorations of the Auditory and Haptic Correlate for the Mobile Device, Proceedings of International Conference on Auditory Display (ICAD 2005), Limerick, Ireland, July 2005.

[16] Reed, C.M., Delhorne, L, and Durlach, N. A Study of the Tactual and Visual Reception of Fingerspelling, Journal of Speech and Hearing Research, 33, December 1990, 786-797.

[17] Tan, H. Z., Perceptual user interfaces: haptic interfaces; Communications of the ACM 43, 3 (Mar. 2000), 40 - 41.

[18] Twitchell, J.B. Twenty Ads that Shook the World. Crown Publishing Group, New York, NY, USA, 2001, pp.62,164-167.

[19] Verrillo, R.T. and Gescheider, G.A. Perception via the Sense of Touch: Chapter 1 from Tactile Aids for the Hearing Impaired, Ed. Summers, Ian). Whurr Publishers, London, 1992.

[20] Russolo, L. The Art Of Noises, Pendragon Press; New York, c1986. 
[21] Gaver, W., Auditory Interface: Chapter from Handbook of Human-Computer Interaction, Eds. Helander, M.G., Landauer, T.K. and Prabhu, P., Elsevier Science; Amsterdam, 1997.

[22] Casey, M.A. (1998). Auditory Group Theory with Applications to Statistical Basis Methods for Structured Audio. MIT PhD Thesis, 1998.

[23] Leplatre, G., Brewster, S.A. (1998). An Investigation of Using Music to Provide Navigation Cues. In Proceedings of ICAD'98 (Glasgow, UK), British Computer Society.

[24] Brewster, S.A. Chapter 12: Non-speech auditory output. In Jacko, J. and Sears, A. (Eds.) The Human Computer Interaction Handbook (Lawrence Erlbaum Associates, USA), 220-239

[25] Blattner, M., Sumikawa, D. \& Greenberg, R. (1989). Earcons and icons: Their structure and common design principles. Human Computer Interaction, 4(1)

[26] Kostka, S. Materials and Techniques of Twentieth-Century Music, Prentice Hall; New Jersey, NJ, 1999.

[27] Yano, H.; Igawa, H.; Kameda, T.; Muzutani, K.; Iwata, H., AudioHaptics: audio and haptic rendering based on a physical model, Proceedings of the Intl. Symposium on Haptic Interfaces for Virtual Environment and Teleoperator Systems (HAPTICS'04), 250- 257.

[28] Bowker, G.,Star, S. Sorting Things Out: Classification and Its Consequences (Inside Technology), MIT Press, Cambridge, MA, 2000, 253.

[29] www.logitech.com

[30] MacLean, K. E. (2000). "Designing with Haptic Feedback", in Proceedings of IEEE Robotics and Automation (ICRA'2000), San Francisco, CA, 2000.

[31] O’Modhrain, S.,Oakley, I. Adding Interactivity: Active Touch in Broadcast Media, Proceedings of the International Symposium on Haptic Interfaces for Virtual Environment and Teleoperator Systems (HAPTICS'04), 293- 294. 